\title{
A Feedback based Probability Selection for Frame forwarding in AVB switched Ethernet
}

\author{
Ang $\mathrm{Gao}^{1}$, Yansu $\mathrm{Hu}^{2 *}$, Lixin $\mathrm{Li}^{1}, \mathrm{Xu} \mathrm{Li}^{1}$ \\ 1. School of Electronics and Information, Northwestern Polytechnical University, \\ Xi'an 710072, China \\ 2. School of Electronics and Control, Chang'an University, Xi'an 710064, China \\ E-mail:gaoang@nwpu.edu.cn;huyansu@163.com
}

\begin{abstract}
In order to support real-time quality of service in time-sensitive and time-tolerant coexisting network in Ethernet, a set of IEEE standards for transporting and forwarding real-time content over Ethernet are proposed that known as Audio Video Bridging (AVB). By bandwidth reservation and priority isolation, AVB traffic are re-mapped into the highest Ethernet frames and are always prior forwarding, so the time delay can be ensured in the specific scope. However, the time-tolerant traffics are simply crude treated by Strict Priority (SP) selection and Best Effort (BE) forwarding. The consequence is that the lowest priorities are over-sacrificed when the bandwidth is limited. In this paper, we proposed a FeedBack based Probability Selection (FBPS) for frame forwarding to enforce a proportional queuing delay. By means of changing the probability of BE traffics been selected, the average queuing delay ration can be controlled in a fixed value. Which ensure high priority BE traffics can enjoy a relatively low latency, and low priority BE can also be operated as well. The experiment demonstrates the effectiveness of FBPS.
\end{abstract}

Keywords: QoS; Ethernet AVB; IEEE802.1Qav; Feedback Control; FeedBack based Probability Selection

\section{Introduction}

As the digital audio and video processing technology has laid a solid technology foundation for the intelligence of automation, avionics and automotive, the demands of standard network technologies for common device are created. Due to the high bandwidth, low cost, easy scalability, high flexibility and IP-based network compatibility, switched Ethernet network for video/audio mixed usage is a common solution. However, multimedia which coexists with traditional control systems will lead to high diversity in size, intensity and timing requirements when the traffic traverses the channel. Adapting standard networks could use compatible technology, but tight quality of service control was difficult [1].

IEEE 802.1 Audio/Video Bridging Task Group (AVB TG) implements a set of protocols on Ethernet which are known as Audio Video Bridging (AVB). AVB implements synchronization services in IEEE 802.1AS [2], Stream Reservation Protocol (SRP) in IEEE 802.1Qat [3] and frame forwarding for AVB traffic in IEEE 802.1Qav [4], where IEEE 802.1AS and IEEE 802.1Qat are the prerequisite for traffic forwarding.

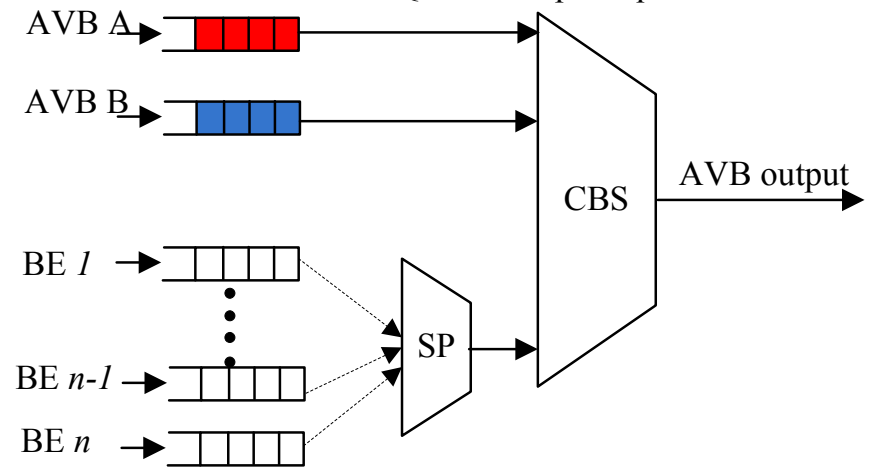

Figure 1. Transmission selection scheme in IEEE 802.1Qav

The IEEE 802.1Qav standard specifies mechanisms to provide guarantees for time-sensitive (i.e. bounded latency and delivery variation), loss-sensitive real-time audio/video data transmission (AV traffic). It separates the network traffic into different traffic classes by using priority mechanism as specified in IEEE 802.1Q. Each traffic uses a dedicated queue with the scheduling of FIFO (First-IN-First-Out) order. As shown in Figure 1, IEEE 802.1Qav supports two transmission selection algorithms in a switched Ethernet network: Credit Based Shaper (CBS) and Strict Priority (SP). 
1). The algorithm defines credits associated to each of the stream reservation (SR) classes (SR-A, SR-B) where a transmission is only allowed when the credits given in bits are greater or equal than zero and no other frames are transmitted at the same time (no conflicting frames). The credits are increased at a rate of idleSlop, when SR classes are waiting for transmission, while decreased at a rate of sendSlop, when SR classes are dequeued and transmitted.

2). SP selection algorithm is used as the default algorithm to select legacy Ethernet frames i.e. Best Effort (BE) frames for transmission. The frames are selected by their priority values. At first, legacy Ethernet frames are selected from the highest non-AVB priority queue until the queue is empty and frames from other non-AVB queues are selected successively based on the priority values.

The initial design of CBS is used to ensure the fair scheduling of the low-priority frames and smooth out the traffic flow to greatly reduce the possibility of dropped packets due to congestion. However, there still exits the over-sacrifice condition of low non-AVB priority. As shown in Figure 2, there are $N=6$ BE traffics and AVB traffics (SR-A and SR-B) in a switched Ethernet network. These different traffics are re-mapped to Ethernet frames by using Virtual Local Area Network (VLAN) tag encoded priority values. All traffics are transmitted on an $8-\mathrm{kHz}$ beat. $20 \%$ bandwidth is reserved for SR-A and SR-B, which is ensured by time synchronization (IEEE 802.1AS) and bandwidth reservation (IEEE 802.1Qat). Legacy Ethernet frames are selected form the highest priority queues.

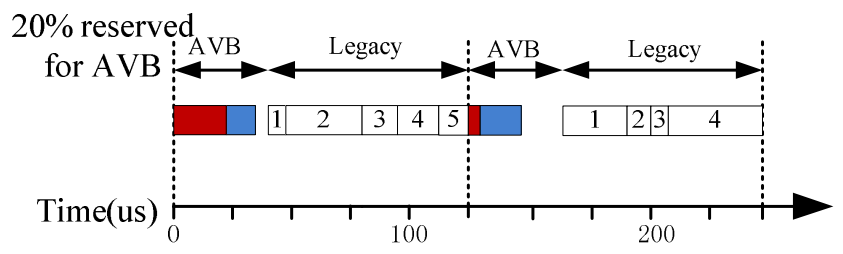

Figure 2. Over-sacrifice of non-AVB low priority frames

In the worst case, a mass of higher BE traffics will block the transmission of lower priority until its queue is empty. For example, the BE frames prioritized 6 in the first and second transition period always have no chance to be transmitted. This phenomenon is named as low priority's starvation.

AVB has become a hot point for the researchers. Lim H T etc. mainly simulated the protocols specified in the IEEE 802.1 AVB standard and compared the performance with the legacy mechanism [5-6]. Paper [7] simulated several cases in order to decide the optimal bandwidth allocation ratio for the scheduled traffic by adjusting scheduled traffic's MTU size. Paper [8] used the shaper context to limit the workload and tighten the worst-case latency metric. Paper [9] presented an approach to mitigate the effect of such interference and reduce the latency for high priority traffic in IEEE 802.1 AVB Networks. However, all the researches focused on the performance analysis and improvement in AVB but without the consideration of the over-sacrifice of low non-AVB priority. In this paper, we propose a probability selection approach based on feedback based control to realize the proportional delay differentiation. Instead of SP algorithm, the approach controls the average delay ratio of BE traffics with different priorities to be a pre-fixed value. On one hand, a desired fixed delay ratio between the BE traffics is differentiated. The higher priority means a lower delay. On the other hand, even the lowest priority traffics still have the chance to be transmitted, which can prevent the over-sacrifice of low priorities.

\section{Probability Selection Architecture and Controller Design}

\subsection{Proportional Delay Differentiation}

Supposing there are $N$ classes of BE traffics in AVB switched Ethernet. The proportional delay differentiation (PDD) scheme is adopted to avoid the over-sacrificed of low priority. The control object is to maintain the actual QoS parameters to meet the Eq. (1).

$$
\frac{\varsigma_{i}}{\varsigma_{j}}=\frac{\delta_{i}}{\delta_{j}}, i=j=1, \cdots N
$$

where $\zeta_{i}$ is the average queuing delay of class $i$ and $\delta_{i}$ is the corresponding priority parameter set by upper layer protocol like re-mapping in IEEE 802.1Q. The class with smaller $\delta$ means a higher priority and expects a lower delay. 
Define $p_{i}(k)$ be the transmission probability of class $i$ at the $k^{\text {th }}$ sampling period and it obeys the constraint of Eq.(2):

$$
\sum_{i=1}^{N} p_{i}(k)=1
$$

Obviously, a class with a higher selection probability waits a shorter queuing time. Meanwhile, the traffics even with the lowest priority always have the chance to be scheduled. So the initial idea of the probability selection control is to realize the PDD guarantees shown as Eq.(1) by adjusting the selection probability of different BE traffic classes.

\subsection{System Modeling}

Figure 3 shows the feedback control model of probability selection architecture in AVB. The output of selection controller is the probability vector which has only $N-1$ independent variables:

$$
X(k)=\left[p_{1}(k), p_{2}(k), \cdots p_{N-1}(k)\right]^{T}
$$

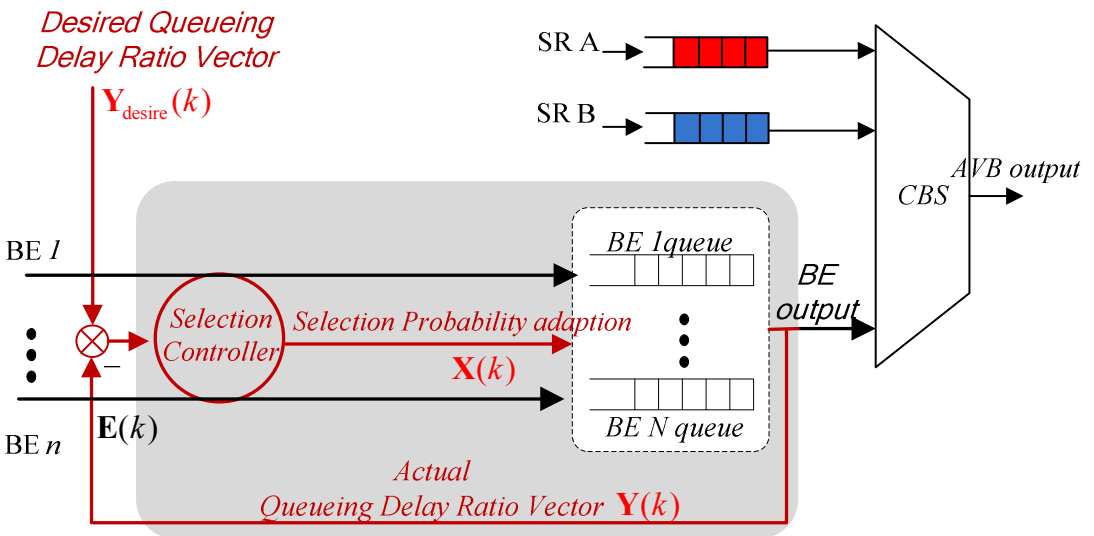

Figure 3. Feedback control model of probability selection architecture

Supposing $y_{i}(k)$ and $y_{i_{\text {desire }}}$ are the normalized delay and desired QoS value respectively according to Eq. (1).

$$
y_{i}(\mathrm{k})=\frac{\varsigma_{i}}{\sum_{l=1}^{N} \varsigma_{l}}, y_{i_{\text {desire }}}=\frac{\delta_{i}}{\sum_{l=1}^{N} \delta_{l}}, 1 \leq i \leq N
$$

Because of $\sum_{i=1}^{N} y_{i}(k)=1$ and $\sum_{i=1}^{N} y_{i_{\text {desire }}}=1$, both the system output vector and the reference input vector also have $O=N-1$ independent variables:

$$
Y(k)=\left[y_{1}(k), y_{2}(k), \cdots y_{N-1}(k)\right]^{T}, Y_{\text {desire }}=\left[y_{1_{\text {desire }}}, y_{2_{\text {desire }}}, \cdots y_{N-1_{\text {desire }}}\right]^{T}
$$

$E(k)$ is the deviation between the measured normalized delay and its desired value.

$$
E(k)=Y(k)-Y_{\text {desire }}
$$

The controller operates by responding to the deviation, i.e. adjusting queuing delay by changing the selection probability. Thus the proportional delay differentiation is sustained. The inherent self-stabilization of feedback mechanism ensures the system good running despite of the uncertainty of traffic flow and interfering data.

The mathematical model can be established based on the variables definition above. Strictly speaking, a discrete and nonlinear model is required for the probability selection architecture. However, such a nonlinear model is not amenable to the straightforward theoretical design and analysis. So the following linear model is used to approximate the system. Supposing r-order could be precise enough, the Z-domain transformation is:

$$
\begin{aligned}
& A\left(z^{-1}\right) Y(k)=B\left(z^{-1}\right) X(k)+\Xi(k) \\
& A\left(z^{-1}\right)=I-A_{1} z^{-1}-\cdots-A_{r} z^{-r}, A_{i} \in R^{O \times O}, 0<i \leq r \\
& B\left(z^{-1}\right)=B_{1} z^{-1}-\cdots-B_{r} z^{-r}, B_{i} \in R^{O \times I}, 0<i \leq r
\end{aligned}
$$

where $\Xi(k)=\left[\varepsilon_{1}(k), \varepsilon_{2}(k), \cdots \varepsilon_{N-1}(k)\right]^{T}$ is $O$-order white noise sequence. Then Eq. (7) can be rewritten as:

$$
\begin{aligned}
& Y(k+1)=\Theta \Phi(k)+\mathcal{E}(k+1) \\
& \Theta=\left[B_{1}, \cdots B_{r}, A_{1}, \cdots A_{r}\right] \in R^{O \times(O \times 2 \times r)}, k \geq r-1 \\
& \Phi(k)=\left[X^{T}(k), \cdots, X^{T}(k-r-1), Y^{T}(k), \cdots, Y^{T}(k-r-1)\right]^{T}
\end{aligned}
$$


We take Recursive Least Square (RLS) algorithm to estimate the parameter matrix $\Theta$ and $F$-test to determinate the system order. The mathematical details can be seen in our previous paper [10].

\subsection{Controller Design}

According to section 2.2, the system transfer function in Z-domain can be described as $G(z)=Y(z) / X(z)$. Supposing the controller transfer function is $D(z)$ and the closed-loop transfer function is:

$$
F(z)=\frac{D(z) G(z)}{1+D(z) G(z)}
$$

Thanks for the Least-Beat Control approach, the system output can follow the input signal in very several sampling time, and can limit the steady-state error to zero (zero steady-state error system). The typical input signal can be described as Eq. (10), such as unit-step function, unit-ramp function and unit of acceleration function.

$$
Y_{\text {desire }}(z)=\frac{A(z)}{\left(1-z^{-1}\right)^{m}}
$$

So the deviation $E$ is

$$
E(z)=F_{e}(z) Y_{\text {desire }}(z)=\frac{F_{e}(z) A(z)}{\left(1-z^{-1}\right)^{m}}
$$

where $F_{e}(z)=1-F(z)$. According to the expiration-value theorem, the system steady-state error is:

$$
E(\infty)=\lim _{z \rightarrow 1}\left(1-z^{-1}\right) E(z)=\lim _{z \rightarrow 1}\left(1-z^{-1}\right) \frac{F_{e}(z) A(z)}{\left(1-z^{-1}\right)^{m}}
$$

If $E(\infty)=0, F_{e}$ must has factor of $\left(1-z^{-1}\right)^{m}$. Take the worst condition i.e. the unit-step function as the input signal which means $m=1$. Put $F(z)=z^{-1}$ into Eq. (9), there is

$$
\frac{D(z) G(z)}{1+D(z) G(z)}=z^{-1}, D(z)=\frac{z^{-1}}{\left(1-z^{-1}\right) G(z)}
$$

\subsection{Probability Selection}

In order to transmit the corresponding frames according to their selection probability $p_{i}(k)$ calculated by the controller, a threshold vector is defined for all the priorities as follows.

$$
\Lambda(k)=\left[\lambda_{0}(k), \lambda_{1}(k), \cdots \lambda_{N-1}(k), \lambda_{N}(k)\right]^{T}, 0=\lambda_{0}(k)<\lambda_{1}(k)<\cdots<\lambda_{N-1}(k)<\lambda_{N}(k)=1
$$

During the BE transmission period, if there is no other BE frames transmitted at the same time, the frames selection is operated as follows:

1). First, generate a random number $s(k)$ submitted to the uniform distribution in the range of [0,1], i.e. $s(k) \square \mathrm{U}(0,1)$;

2). Second, compare $s(k)$ with the threshold vector. If $\lambda_{i-1}<s(k)<\lambda_{i}, i=1,2, \cdots N$, then the package from the $i^{\text {th }}$ priority queue is transmitted;

3). It is intuitional to draw the value of threshold parameters:

\section{Experiments and Results}

$$
\left\{\begin{array}{l}
p_{1}(k)=\lambda_{1}(k) \\
p_{2}(k)=\lambda_{2}(k)-\lambda_{1}(k) \\
\vdots \\
p_{N-1}(k)=\lambda_{N-1}(k)-\lambda_{N-2}(k)
\end{array}\right.
$$

\subsection{Configuration}

We take simulation to test the performance of FeedBack based Probability Selection (FBPS) algorithm. AVB Switcher forwards the prioritized Ethernet frames. The total output bandwidth of switcher is $100 \mathrm{Mbps}$, and $20 \%$ bandwidth is reserved for AVB and the transmission is $8 \mathrm{kHz}$ beat. Besides AVB traffics, there are other three kinds of BE traffics, which are marked as BE 1, BE 2 and BE 3. Define BE 1 be the highest priority that must be transmitted first by SP. Because AVB traffics and BE traffics are isolated strictly by bandwidth reservation, so we only analysis the feature of BE traffics in the following simulation. In all the BE traffics, the Ethernet payload size submits the Pareto distribution. The maximum payload is 1500 byte and the average length is 700 byte. The 
depth of all the BE queues is 1000 frames.

Considered the worst case:

1). The arriving rate of the highest priority (BE 1) changes between 400-1200 frames/second with the period of $600 \mathrm{~s}$;

2). The arriving rate of the medium priority (BE 2) is nearly constant at 600 frames/second;

3). The lowers priority (BE 3) changes with the same period but opposite phase of BE 1 .

The experiment operates last 2000s and the selection controller starts at about $1000 \mathrm{~s}$. The period changed traffics are equivalent to positive and negative step-function signals for a control system, which is theoretically the worst case for a controller.
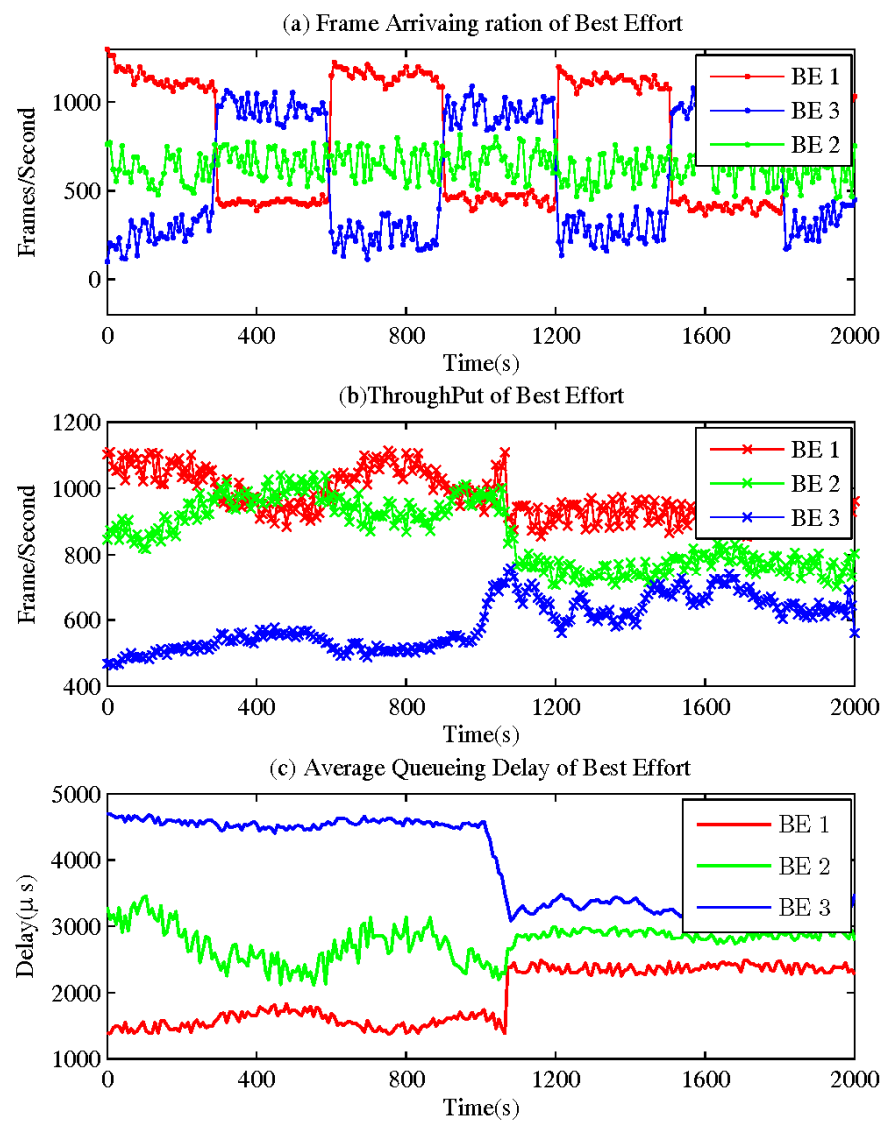

Figure 4. Throughput and Queuing Delay of BE traffics in FBPS

\subsection{Results}

The results are shown in Figure 4.

In the former $1000 \mathrm{~s}$, original SP of AVB is adopted for the BE traffics transmission.

1). Because BE 1 traffic frames are always firstly filled in the legacy bandwidth, so the throughput of BE 1 allows the tendency of the arriving rate and queuing delay is relative small;

2). The throughput and delay of BE 1 show a little fluctuates because of the changing queuing length caused by the arriving rate, which will in turn influence the throughput and delay;

3). Although the arriving rate of BE 2 is nearly constant, the throughput changing with BE 1 but in opposite phase. This is because the legacy bandwidth can be used by BE 2 only after the transmission of BE 1 frames. Bandwidth is fixed, so throughput of BE 2 fluctuates with BE 1 arriving rate. The average delay of BE 2 also shows the same phenomena with throughput. The higher throughput responds to the lower delay.

4). The throughput of BE 3 is low and correspondingly the delay is high no matter how the arriving rate changes. It is because the former two traffics (BE 1 and BE 2) have occupied most of the bandwidth, 
and there is nearly no chance for BE 3 transmission (the starvation of BE 3 ).

FBPS operates after 1000s. The desired queuing delay ratio is set to be $\delta_{1} / \delta_{2} / \delta_{3}=2 / 3 / 4$

1). The analysis of BE 3 shows that FBPS can effectively inhibit the over-sacrifice of low priority, and supports the fairness of BE traffics. Although the cost of the fairness is the performance decrease (delay and throughput) of other higher BE classes, the QoS for all BE traffics can be guaranteed.

2). The average queuing delay ratio can converge to a constant value as we set before. This illustrates the validity of the FBPS algorithm. Meanwhile, the controller presents a better dynamic performance, i.e. setting time and overshoots which is accordant to the theory design in Section 2.3.

3). It is worth mention that the system is always stable although the arriving rate of BE 1 and $\mathrm{BE} 2$ keeping step changes. This proves again that FBPS has a great robustness because of the feedback scheme.

\section{Conclusion}

As the consumer demands for multimedia applications of Ethernet have been huge increased which draws higher requests in the network bandwidth and quality of service (QoS), AVB has been regarded as the feasible technology for the next generation. It is completely compatible with the existing Ethernet scheme. At the same time, by the guarantee of bandwidth, latency and time synchronization, Ethernet AVB provides perfect QoS to support different kinds of multimedia applications. Different with other researches which mainly focus on the SR traffics, we try to find an approach to support the QoS of BE traffics. The feedback based probability selection algorithm is proposed in this paper to realize the proportional delay differentiation in BE traffics, and the experiments show the validity and robustness. On one hand, a desired fixed delay ratio between the BE traffic is differentiated. On the other hand, even the lowest priority traffics still have the chance to be transmitted, which can prevent the over-sacrifice of low priorities.

\section{Acknowledgements}

This work is supported by Shaanxi Province natural science basic research plan surface project (No. 2016JM6062), by China Aerospace Science and Technology Corporation Aerospace Science and Technology Innovation Fund funded project.

\section{References}

[1] Lim H T, Weckemann K, Herrscher D. Performance study of an in-car switched ethernet network without prioritization. International Workshop on Communication Technologies for Vehicles, Springer Berlin Heidelberg, 2011: 165-175.

[2] IEEE Std 802.1As, IEEE Standard for Local and metropolitan area networks-Timing and Synchronization for TimeSensitive Applications in Bridged Local Area Networks, November 2010.

[3] IEEE Std 802.1Qat, IEEE Standard for Local and metropolitan area networks, Virtual Bridged Local Area Networks, Amendment 14: Stream Reservation Protocol, September 2010.

[4] IEEE Std. 802.1Qav, IEEE Standard for Local and metropolitan area networks, Virtual Bridged Local AreaNetworks, Amendment 12: Forwarding and Queuing Enhancements for Time-Sensitive Streams, January 2010.

[5] Lim H T, Herrscher D, Waltl M J, et al. Performance analysis of the IEEE 802.1 ethernet audio/video bridging standard[C]. Proceedings of the 5th International ICST Conference on Simulation Tools and Techniques. ICST (Institute for Computer Sciences, Social-Informatics and Telecommunications Engineering), 2012: 27-36.

[6] Lim H T, Herrscher D, Chaari F. Performance comparison of ieee $802.1 \mathrm{q}$ and ieee 802.1 avb in an ethernet-based invehicle network. Computing Technology and Information Management (ICCM), 2012 8th International Conference on. IEEE, 2012, 1: 1-6.

[7] Ko J, Lee J, Park C, et al. Research on optimal bandwidth allocation for the scheduled traffic in ieee 802.1 AVB. 2015 IEEE International Conference on Vehicular Electronics and Safety (ICVES). IEEE, 2015: 31-35.

[8] Axer P, Thiele D, Ernst R, et al. Exploiting shaper context to improve performance bounds of ethernet avb networks. Proceedings of the 51st Annual Design Automation Conference. ACM, 2014: 1-6.

[9] Imtiaz J, Jasperneite J, Weber K. Approaches to reduce the latency for high priority traffic in IEEE 802.1 AVB networks. Factory Communication Systems (WFCS), 2012 9th IEEE International Workshop on. IEEE, 2012: 161-164.

[10] Gao A, Hu Y. A Feedback Approach for QoS-Enhanced MAC in Wireless Sensor Network. Journal of Sensors, 2016,1:1-12 